\title{
Comparación del comportamiento de dos escayolas amasadas con aguas contaminadas
}

Fecha de recepción: 17/11/87

\author{
E. PERIS MORA. Prof. Tit. (Ing. de la Constr.) \\ M. C. MILLAN GONZALEZ. Prof. Tit. (Física) \\ M. BONILLA SALVADOR. Prof. Ate. (Ing. de la Constr.) \\ UNIVERSIDAD POLITECNICA DE VALENCIA
}

\section{INTRODUCCION}

Las investigaciones que se publican actualmente acerca de la físico-química del yeso como material de construcción resultan relativamente escasas si se tiene en cuenta que, pese a tratarse de un material conocido y utilizado desde hace milenios, todavia hoy se ignoran algunos aspectos de su comportamiento.

Se trata no obstante de un material digno de interés en nuestro tiempo y circunstancia, como prueban algunos datos económicos correspondientes al período 1978-81 del que se dispone información. Mientras que el número de empresas españolas dedicadas a la fabricación de materiales de construcción en "hormigón, cemento, yeso y escayola" pasó de 4.785 a 4.242, probablemente como consecuencia de la crisis del sector de la construcción que redujo el número de proyectos visados por los Colegios de Arquitectos en un $23 \%$; al yeso no le afectaba. Se aumentaba la producción de "yesos, escayolas y tiza" en un $24 \%$ mientras que la mayoría de los restantes materiales de construcción descendió notablemente en sus producciones. Se trata, como vemos, de un producto que soporta relativamente bien la situación de crisis posiblemente porque a sus relativas facilidades de producción - abundancia en España, costos relativamente reducidos de combustibles en razón de temperaturas moderadas de fabricación, etc. - así como a la versatilidad de sus aplicaciones - albañileria, prefabricados, aplicaciones en restauración y acondicionamiento de edificios-. En especial se debe destacar la adaptabilidad al trabajo de pequeñas empresas de prefabricación, de tipo casi-artesanal o familiar que son bastante resistentes a las situaciones de debilidad en el sector de la construcción.
Los yesos y escayolas producidos en España se obtienen de los minerales naturales (unos 5,3 millones de tonaladas/año de yeso crudo y anhidrita en el período 78/81). Hoy no parece aconsejable la opción de recuperar los residuos ricos en yeso como son, por ejemplo, los efluentes de centrales térmicas que utilizan lignitos como combustibles y que producen grandes cantidades de óxido de azufre. Si bien los criterios económicos todavía no justifican la recuperación, es deseable el limitar las emisiones cuanto antes, con lo que se plantea la necesidad de buscar alternativas de uso para los sulfatos residuales. La calidad de los yesos y escayolas producidas en nuestro país está regulada por las normas UNE así como por el "pliego general de condiciones para la recepción de yesos" (MOPU, 70). Si bien la existencia de esas reglamentaciones resulta una garantía en la interpretación de criterios de calidad, en ocasiones las condiciones contempladas en esas normas no dan solución a todas las demandas que sobre esos materiales pueden hacerse.

\section{DEFINICION DEL PROBLEMA}

Estudiamos en el presente trabajo dos escayolas de diferente procedencia (que llamaremos $M$ e l) y que vienen siendo utilizadas en la fabricación de placas de gran esbeltez. Se utiliza en esa fabricación un procedimiento automatizado de pesada, dosificación, enmoldado, etcétera, de manera que el factor "sistema de fabricación" no explica el diferente comportamiento entre los dos materiales. Mientras que la escayola de la clase M produce placas satisfactorias en todos los casos, las fabricadas con el material I al cabo de poco tiempo presentan comportamiento viscoso, es decir: sufren de- 
formaciones lentas, curvándose si son puestas en obra; en otros casos al quedar almacenadas en fábrica presentan alabeamientos sobre las mismas jaulas de almacenaje quedando inservibles. Esto sucede a pesar de que el material ha sido sometido a controles de calidad, de acuerdo cori Norma y Pliego y superado dichos controles. La aparición del comportamiento viscoso en las piezas puestas en servicio ocasiona, además, como es natural, graves consecuencias económicas, así como el deterioro de imagen de la marca.

En el estudio emprendido se propuso la interpretación del fenómeno de la fluencia de las placas, de manera que pudiera ser propuesta la solución tecnológica adecuada.

\section{MATERIALES}

Se parte de escayolas de diferente procedencia que son utilizadas para fabricar placas en un proceso automático. En la elaboración se emplea $8,2 \mathrm{~kg}$ de escayola, $6,5 \mathrm{~kg}$ de agua de amasado además de la cantidad adecuada de fibras de vidrio (E.C. 11.2400) ISO 2087 que constituye el reforzante para el trabajo a flexión; se aplica asi mismo una pequeña cantidad de desencofrante sobre el molde, de caucho.

El análisis químico de los materiales proporciona los resultados siguientes:

\begin{tabular}{lrrr} 
& Escayola M & & Escayola I \\
\cline { 2 - 2 } $\mathrm{SO}_{3}$ UNE 102-032-84 & 51,12 & & $51,00 \%$ \\
Res. insol. UNE 102-032-84 & 4,75 & & $4,25 \%$ \\
$\mathrm{CaO}$ UNE 102-032-84 & 34,40 & & $35,95 \%$ \\
$\mathrm{R}_{2} \mathrm{O}_{3}$ UNE 102-032-84 & 0.55 & & $0,68 \%$ \\
$\mathrm{Cl}^{-}$UNE 102-032-84 & 8,08 & $0,07 \%$ \\
$\mathrm{CaCO}_{3}$ Calcimetro (Bernard) & 0,78 & $0,78 \%$ \\
MgO (ABS. AT.) & 0,83 & $2,28 \%$
\end{tabular}

Las aguas utilizadas para el amasado tienen una composición incierta. Se utiliizó agua procedente de la red de distribución que presenta composición variable, si bien en general debe considerarse agua de mala calidad. Al proceder de mezcla de aguas superficiales y subteráneas en función de las demandas, puede variar en diferentes épocas del año y aun en días consecutivos. Ocasionalmente se usó agua de aljibe de mejor calidad la cual, según los fabricantes, proporcionó placas satisfactorias con ambas escayolas; no obstante la pequeña capacidad del allibe no permite abaste. cer las necesidades de labricación más que en muy pocos casos. Las composiciones que indicamos a continuación tienen solamente carácter orientador en 10 que se refiere a interpretar el fenómeno que se estudia:

\begin{tabular}{lccccc} 
& Agua de red & & \multicolumn{2}{c}{ Agua de aljibe } \\
\cline { 2 - 3 } $\mathrm{NH}_{3}$ & 0,06 & & 0,05 & $\mathrm{mg} / \mathrm{l}$ \\
$\mathrm{NO}_{2}{ }^{-}$ & 0,08 & & 0,08 & $\mathrm{mg} / \mathrm{l}$ \\
$\mathrm{NO}_{3}{ }^{-}$ & 93,0 & & 7,0 & $\mathrm{mg} / \mathrm{l}$ \\
M.O. $\left(\mathrm{mg} \mathrm{O}_{2} / \mathrm{l}\right)$ & 4,2 & & 1,0 & $\mathrm{mg} / \mathrm{l}$ \\
Dureza & 257 & & 11,2 & grados Franc. \\
$\mathrm{Cl}^{-}$ & 1.200 & & 20,0 & $\mathrm{mg} / \mathrm{l}$
\end{tabular}

\section{DISCUSION Y ENSAYOS COMPLEMENTARIOS}

El estudio de la información inicial, obtenida al caracterizar los materiales escayola y agua, no proporciona criterios suficientes para justificar el diferente comportamiento en servicio de las placas. Destacan, sin embargo, las diferencias en los contenidos en $\mathrm{Mg}$ - mayores en los materiales de la clase 1- así como los elevados valores de las concentraciones en $\mathrm{Cl}^{-}$y $\mathrm{NO}_{3}^{-}$ en las aguas de la red de distribución. Es de señalar que en las investigaciones realizadas por Ridge (7) y (8) referidas a la comparación de yesos de diferente procedencia, también aparecen comportamientos anómalos en ambas para los materiales que presentan altos contenidos en $\mathrm{Mg}$; el autor, sin embargo, no explica en ninguna de las dos publicaciones la posible relación.

Por otra parte los datos que estas tablas se contienen no hacen sino corroborar que las escayolas poseen la calidad química adecuada de acuerdo con las normas, por más que en el trabajo a que se las somete les haga responder de manera no prevista en los ensayos. Otras propiedades de los materiales son las siguientes:

$\begin{array}{lcc}\begin{array}{c}\text { Finura de molido } \\ \text { (UNE 102-011-83) }\end{array} & \text { Escayola M } & \text { Escayola I } \\ \begin{array}{c}\text { Amasada a saturacion } \\ \text { (UNE 102-031-82) }\end{array} & 0,20 & 0,35 \% \\ \text { Principio/fin fraguado } & 0,70 & 0,65 \\ & 7^{\prime} / 23,5^{\prime} & 7^{\prime} / 21,5^{\prime}\end{array}$

La ausencia de criterios diferenciadores suficientes desde el punto de vista quimico suciere la necesidad de investigar si son los onsta. les de ambos materiales los que justifican la diferencia. A ese efecto es preciso comprobar si entre las dos escayolas la proporción entre las formas alfa y beta es distinta. Como es sabido ambas formas no corresponden a sis" temas cristalinos diferentes (y por lo tanio son indistinguibles mediante difractometria de rayos $X$ ) sino que se trata de dos grados de cristalinidad. Esto implica diferentes superfi" 
cies específicas y consecuentemente resistencias mecánicas muy diversas para los materiales ricos en forma alfa o beta. Muchos aútores mantienen la denominación (3) a pesar de la interpretación equívoca a la que se presta desde el punto de vista habitual de las ciencias del estado sólido, por eso se ha sugerido a veces (2) la conveniencia de sustituir las denominaciones por las de alfa y alfa prima respectivamente.

En nuestras muestras se aplicó la metodología tradicional (5) para diferenciar ambas formas mediante observación microscópica, no obteniendo resultados concluyentes, lo que por otra parte era de esperar dadas las resistencias mecánicas de ambos materiales en ensayos de flexotracción. La escayola I cuyo comportamiento en servicio resulta problemático ofrecía valores muy semejantes, y en ocasiones superiores, a la otra; ambas satisfacen las exigencias del pliego.

La difractometría de R-X (Fig. 1) más que proporcionar criterios de diferenciación ofrece una imagen de gran similitud, tanto entre las escayolas como entre los materiales resultantes al fabricar las placas.

Al recurrir a la observación microscópica, mediante microscopía electrónica de barrido, aparecen muy claras las semejanzas entre las escayolas, pero también diferencias entre las placas construidas con ellas (fotos 1 y 2). Así se encuentra que los hábitos cristalinos de la placa I se manifiesta como un grano de granulometría dispersa, frente a granos muy uniformes de tamaño y forma en agujas en la placa $M$. Efectivamente los granos más redondeados de la placa I parecen ser más favorables al comportamiento viscoso que los granos en forma de aguja.

A partir de este punto de la investigación se recurre a completar el estudio con la realiza-

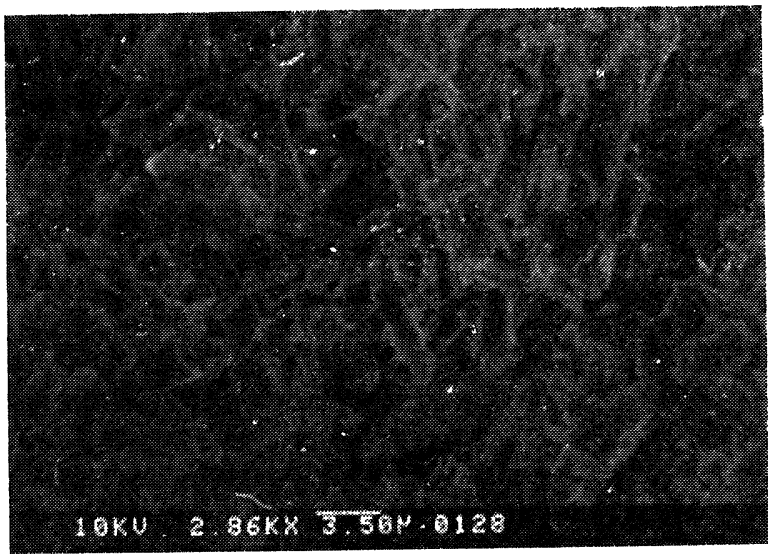

Foto 1.-Placa escayola I. ción de ensayos tecnológicos que reproducen en alguna medida las condiciones de trabajo que sufren los materiales en servicio. El primero de ellos consiste en medir las variaciones en la deformación (flecha) de una de las placas deformadas. La placa tiene una flecha de $13 \mathrm{~cm}$ y se sitúa apoyada sobre una superficie horizontal, plana, por su parte cóncava; no se controla la humedad ambiental, que oscila entre el 69 y el $74 \%$ durante los días del ensayo. Al cabo de 5 dias la flecha ha dismi-

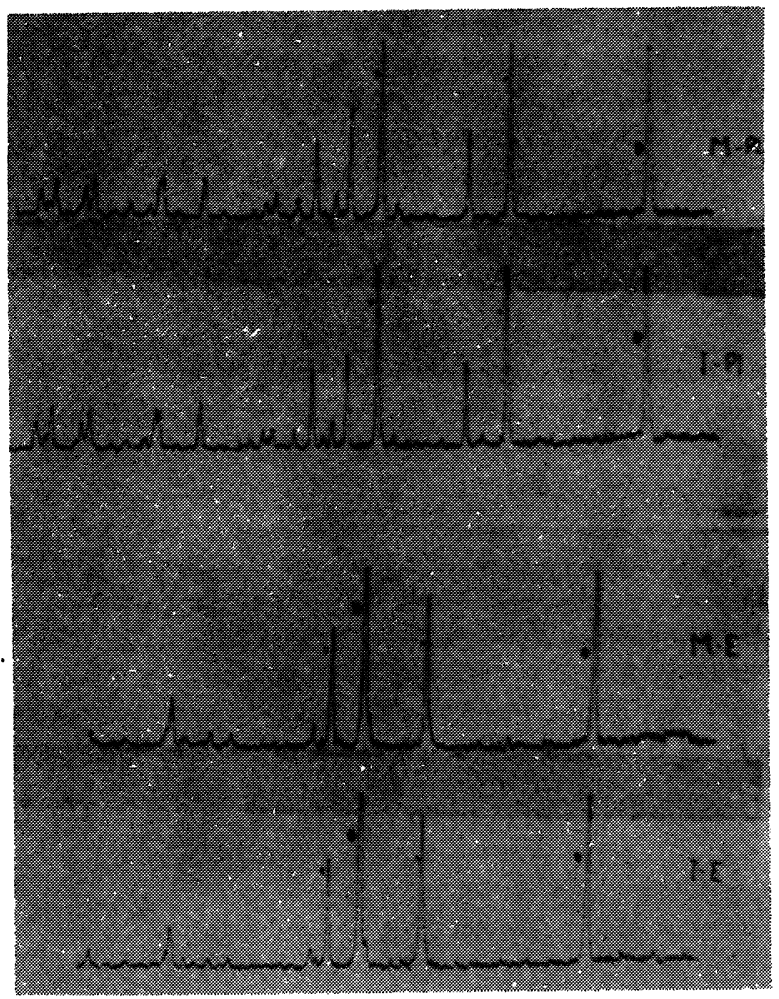

Fig. 1.-Difractogramas de R-X correspondientes a las escayolas (I-E y M-E) y al material de las placas (I-P1 y M-P1). Se señalan los picos de máxima intensidad del hemihidrato y dihidrato, mediante flechas de diferente tamaño $\left(1 / I_{0}\right.$ mayor del $50 \%)$.

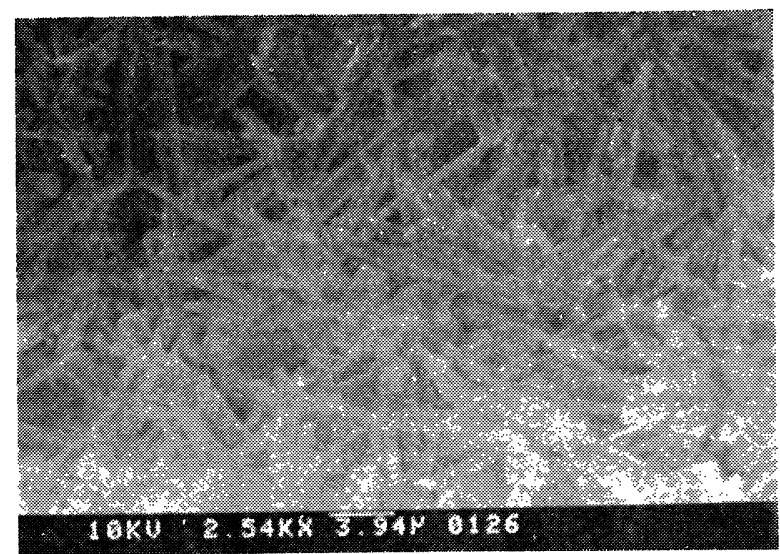

Foto 2.-Placa escayola M. 
nuido a 4,2 cm; después de 9 dias ha desaparecido por completo al asentar la placa sobre el plano.

Simultáneamente se llevan a cabo otras experiencias, en este caso con control de humedad. En ellas una pareja de placas de los dos materiales que se comparan se mantienen apoyadas por sus extremos -luz de $115 \mathrm{~cm}-$ y se conservan en:

a) Cámara húmeda; temperatura de $25^{\circ} \mathrm{C} \mathrm{y} \mathrm{Hr}$ mayor del $95 \%$.

b) Cámara seca; temperatura $30^{\circ} \mathrm{C}$ aprox. $\mathrm{Hr}$ menor del $60 \%$.

Los resultados de la experiencia (fotos 3 y 4) muestran que mientras en la cámara húmeda ambas placas sufren fractura a las pocas horas del inicio, rompiendo con flecha próxima a $10 \mathrm{~cm}$, las placas de la cámara seca, por el contrario, no experimentan aumento de la deformación con el tiempo ni, consecuentemente, fractura. Si bien en las primeras horas la placa I adopta una deformación mayor que la M (ésta de carácter elástico) el paso del tiempo no produce fenómenos de aumento de flecha durante cuatro semanas que se mantiene el ensayo. Transcurridas éstas, al abandonar el control de la humedad y temperatura se produce deformación y rotura.

Los ensayos anteriores permiten atribuir el fenomeno viscoso a la presencia de humedad, entre otras causas. Además existen razones para atribuir a los dos materiales diferente capacidad de retenerla. La presencia de iones $\mathrm{Cl}^{-}$y $\mathrm{NO}_{3}{ }^{-}$procedentes de las aguas de amasado, además de la mayor proporción de magnesio en la escayola que ofrece comportamiento problemático, parece ser la razón principal del diferente comportamiento entre ambas. La difractometría de $R-X$ realizada anteriormente no proporciona evidencia de la pre-

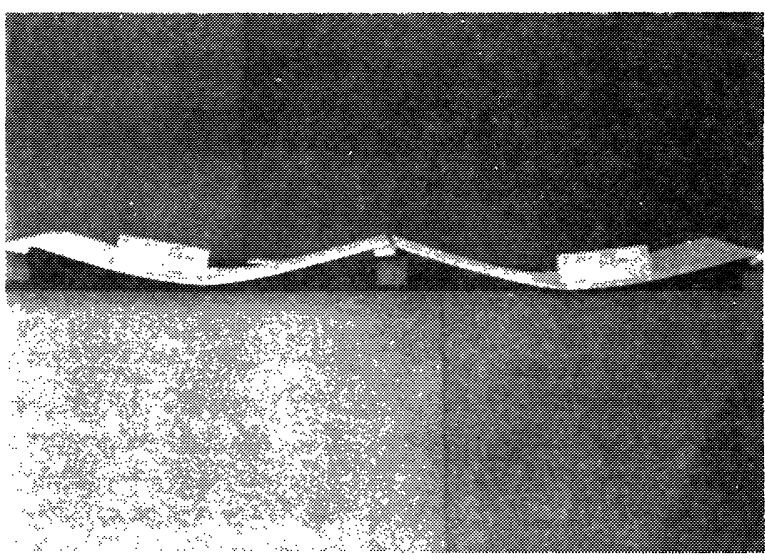

Foto 3.--Placas $\mathrm{M}$ e I conservadas en cámara húmeda. Al poco tiempo de iniciarse el ensayo se deforman y rompen.

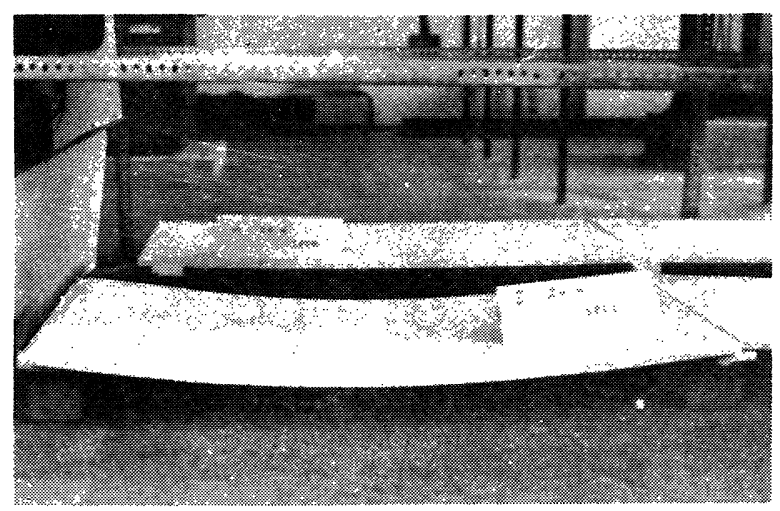

Foto 4.-Conservadas en cámara "seca" soportan la posición incluso durante 4 semanas. La deformación de la placa I se produce inmediatamente de iniciado el ensayo (conservando la placa algo de humedad).

sencia de las sales higroscópicas $\mathrm{MgCl}_{2} \mathrm{ni}$ $\mathrm{Mg}\left(\mathrm{NO}_{3}\right)_{2}$ pues la técnica no es capaz de detectar concentraciones tan pequeñas como las que podrían darse aquí. No obstante tal hipótesis es razonable. La posible presencia de esas sales sería debida, en parte, al aporte de la propia placa y, en parte, a los aportes del agua de amasado utilizada. Consideramos al $\mathrm{Cl}^{-}$como el principal responsable, en su caso, del fenómeno ya que, tanto por su mucho más alta concentración, como por su influencia en los procesos de fraguado, provoca efectos más notables.

Además del carácter higroscópico que puede aportar a la placa, el $\mathrm{Cl}^{-}$modifica las condiciones del fraguado y consecuentemente de los cristales que se forman durante el mismo (6) (8) (1). Algunas experiencias que se describen a continuación se llevaron a la práctica para interpretar en qué medida ambas escayolas eran susceptibles de reacción frente a este contaminante.

En la determinación de las resistencias a flexotracción y compresión de probetas amasadas con aguas contaminadas, se realizaron tres amasadas respectivamente con agua destilada, agua de red $\left(1.200 \mathrm{mg} / \mathrm{l} \mathrm{de} \mathrm{Cl}^{-}\right)$y agua fuertemente contaminada con $\mathrm{Cl}^{-}(3.000 \mathrm{mg} / \mathrm{l})$. Se obtiene el conjunto de resultados siguientes:

\begin{tabular}{|c|c|c|c|c|}
\hline & \multicolumn{2}{|c|}{ Escayola M } & \multicolumn{2}{|c|}{ Escayola I } \\
\hline Destilada & $\begin{array}{c}\text { Flexotr. } \\
30\end{array}$ & $\begin{array}{c}\text { Comp. } \\
86\end{array}$ & $\begin{array}{c}\text { Flexotr. } \\
28\end{array}$ & $\begin{array}{c}\text { Comp. } \\
76 \mathrm{~kg} / \mathrm{cm}^{2}\end{array}$ \\
\hline Red & 26 & 71 & 25 & $83 \mathrm{~kg} / \mathrm{cm}^{2}$ \\
\hline Contam. $\mathrm{Cr}^{-}$ & 33 & 83 & 38 & $92 \mathrm{~kg} / \mathrm{cm}^{2}$ \\
\hline
\end{tabular}

Los resultados anteriores ofrecen la peculiaridad de que, tratándose de pruebas de corta duración, la presencia de $\mathrm{Cl}^{-}$(aceleradores de fraguado) proporciona una elevación de las re- 
sistencias en la escayola de la que sabemos se comporta peor en esfuerzos sostenidos durante largos períodos; entonces puede sufrir deformación viscosa. Hay que hacer notar, sin embargo, que de acuerdo con la norma, las probetas ensayadas han sido sometidas a secado en estufa durante las horas que preceden al ensayo de flexotracción.

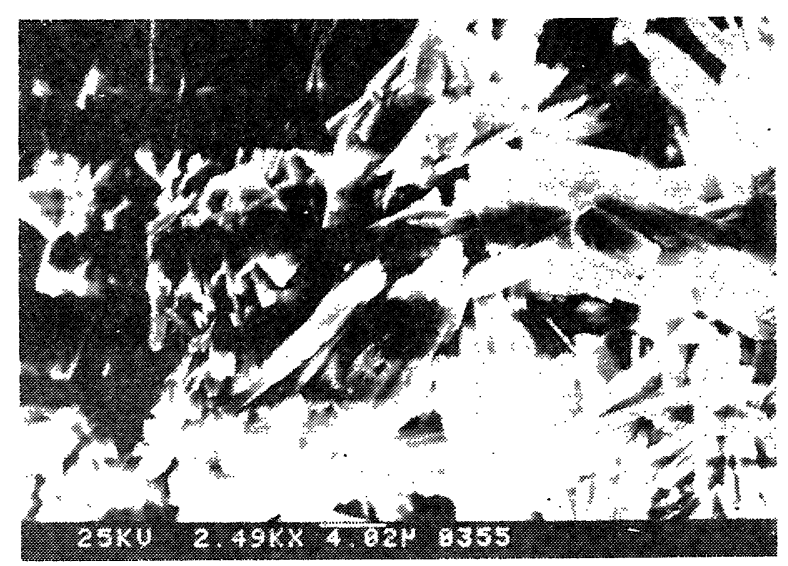

Foto 5.-Escayola M. Agua destilada.

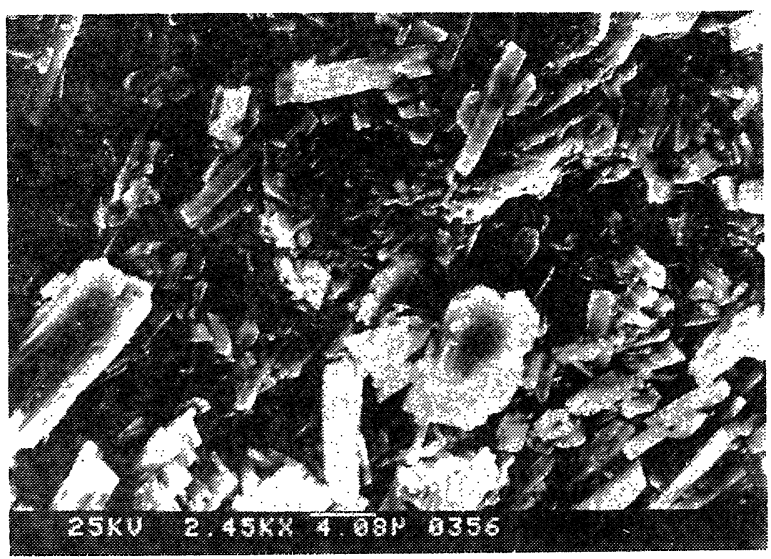

Foto 6.-Escayola I. Agua destilada.

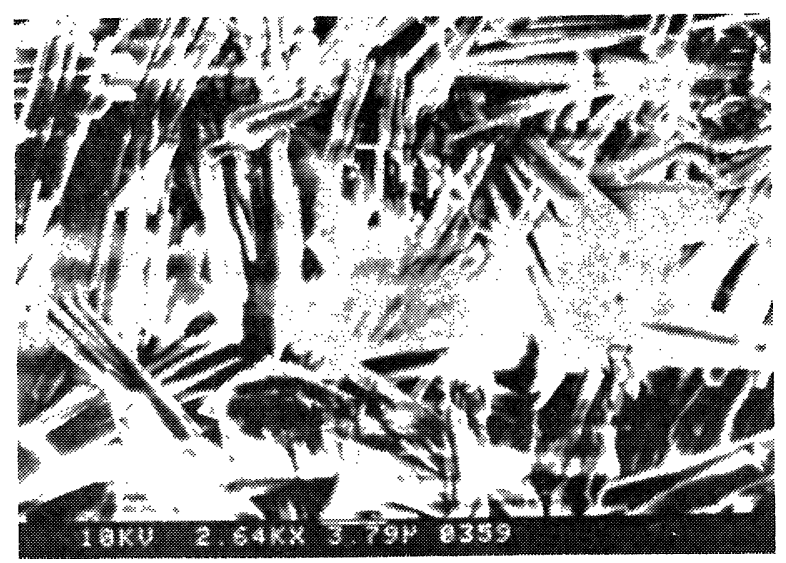

Foto 7.--Escayola M. Agua de red.
Al realizar una observación microscópica de los amasados de ambas escayolas con los diferentes tipos de agua se descubre además que el efecto de la contaminación por cloruros es mucho más manifiesto en la escayola I, de peor comportamiento en servicio (fotos 5 y siguientes).

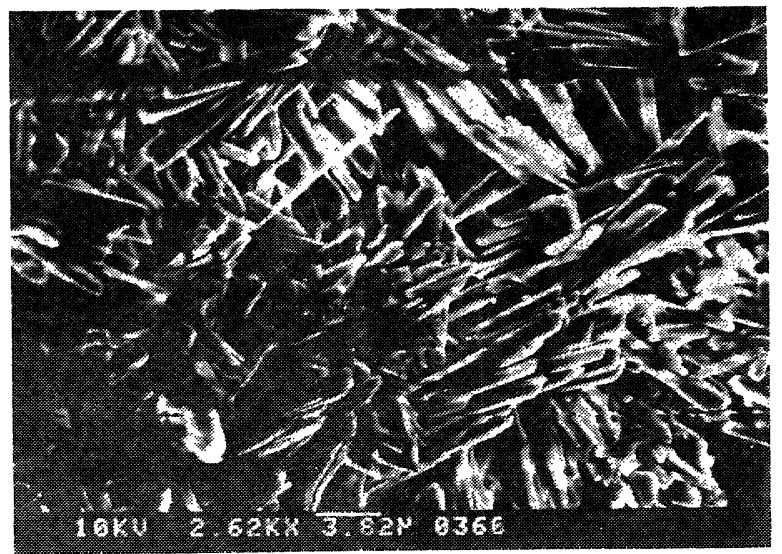

Foto 8.-Escayola I. Agua de red.

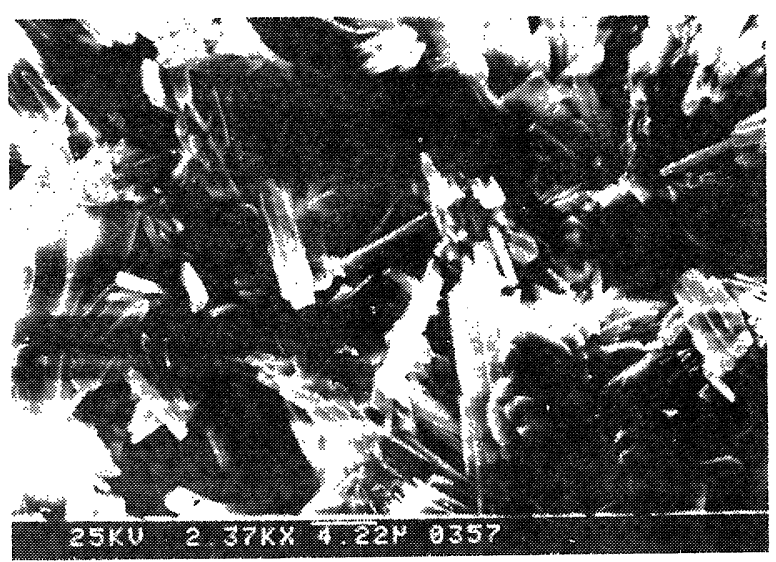

Foto 9.-Escayola M. Agua contaminada $\mathrm{Cl}^{-}$.

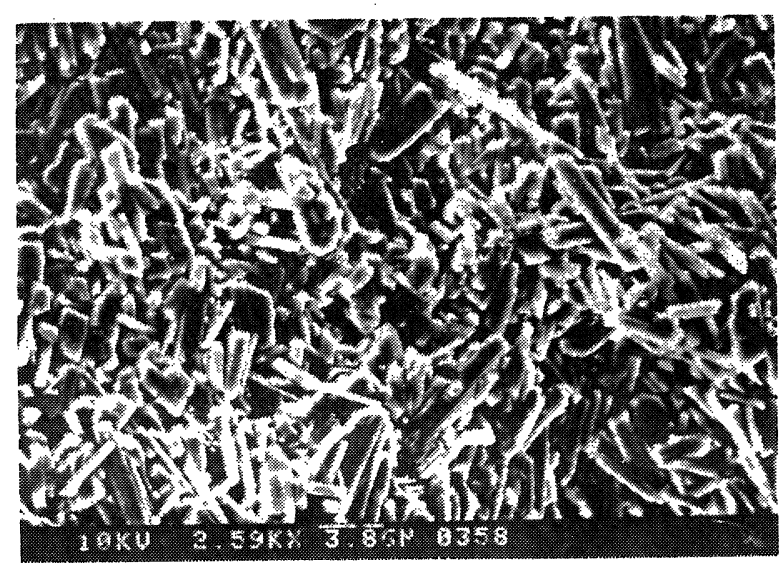

Foto 10.-Escayola I. Agua contaminada $\mathrm{Cl}^{-}$ 
Fueron realizadas también otras pruebas de carácter complementario. Una de ellas consistió en comprobar que las placas fabricadas con la escayola de tipo $\mathrm{M}$, de comportamiento satisfactorio, se convertía en higroscópica cuando se le impregna con una disolución de $\mathrm{MgCl}_{2}$ en concentración equivalente al mayor contenido en $\mathrm{Mg}$ de la otra. De esa manera, pese a que esa contaminación no alcanza a provocar transformaciones en la textura cristalina, se favorece la deformación por el aumento del carácter higroscópico de la placa. Otros ensayos consistieron en la producción de placas en el laboratorio, manualmente, en donde se apreció la mayor respuesta al ion $\mathrm{Cl}^{-}$de la escayola del tipo I.

\section{CONCLUSIONES}

\section{Primera}

Se han realizado una serie de experiencias que permiten comparar e interpretar el diferente comportamiento de dos escayolas amasadas con aguas contaminadas fundamentalmente con ion $\mathrm{Cl}^{-}$. Se ha emprendido este estudio porque, pese a tratarse de dos materiales que cumplen las especificaciones del Pliego y demás normas, entre ellas aparece una diferencia importante en el comportamiento cuando son utilizadas para fabricación de placas de gran esbeltez. En el caso que nos ocupa las placas fabricadas con una de las dos escayolas resultaban totalmente inservibles, lo que daba lugar a importantes pérdidas económicas. La otra escayola, sin embargo, proporcionaba placas satisfactorias utilizando idénticas tecnologías de fabricación y componentes análogos.

\section{Segunda}

Se sugiere como hipótesis que la presencia de $\mathrm{Cl}^{-}$contaminante del agua de amasado origina, sobre todo como consecuencia de la mayor concentración de $\mathrm{Mg}$ que tiene la escayola problema, la deformación del $\mathrm{MgCl}_{2}$, muy higroscópico, que favorece la disminución de resistencias de las placas en servicio, pese a los aceptables resultados de las resistencias en ensayos normalizados. Además del efecto higroscópico, la presencia del ion $\mathrm{Cl}^{-}$en las aguas originaría importantes transformaciones en los hábitos cristalinos del dihidrato cálcico formado al fraguar. No resulta posible la confirmación de esta hipótesis, pues las técnicas analíticas disponibles no son capaces de detectar la presencia del cristal $\mathrm{MgCl}_{2}$ en las proporciones en que aparecen.

\section{Tercera}

Los resultados sugieren la necesidad de complementar los ensayos habituales a que se someten estos materiales en su recepción normal, con otros ensayos tecnológicos y físicoquímicos, ya que, como se ha visto en nuestro estudio, dichos ensayos resultaban insuficientes para dar un material como satisfactorio.

\section{BIBLIOGRAFIA}

(1) BRANDSE, W.P.; ROSMALEN, G.M. Van: The influence of sodium Chloride on the crystalization rate of gypsum. Inorg. Nucl. Chem. 1977. Vol. 39, pp. 2007-2010.

(2) CONSIDIM, D.M.: Chemical and Process Technology Encycl. Mc Graw Hill, 1974.

(3) COLUSSI, I.; LONGO, V.: Applications of Gypsum in building. II Cemento, 1974. Vol. 4, pp. 187-214.

(4) INE: Anuario estadístico 1985.

(5) Importance de la composition des Platres industriels: Bull. Soc. Fr. Ceram. Vol. 67 pp. 21-35. 1976.

(6) MIKHAIL, R. Sh.; MALEK, R.I.A.: Microstructure of Hardened Gypsum Pastes. J. Appl. Chem. Biotechnol. Vol. 21, Oct. 1971.

(7) RIDGE, M.J.; GOEL, G.R.: Physical properties of calcined gypsum. J. Appl. Chem. Vol. 12, pp. 437-444. Oct. 1962.

(8) RIDGE, M. J.; KING, G.A.; MOLONY, B.C.: Variations in gypsum and the reactivity of its product of calcination. J. Appl. Chem. Biotechnol. Vol. 25. pp. 289-295, 1975. 